\title{
Automatically presented groups
}

\author{
Anna Erschler
}

\begin{abstract}
We introduce the notions of automatically presented groups and piecewise automatically presented groups. We show that if $G$ is a piecewise automatically presented group satisfying the property $T$ of Kazhdan, then $G$ is finite. We prove that if $G$ is amenable and finitely presented, then $G$ is virtually abelian. We give further restrictions for a group to be piecewise automatically presented and study properties of such groups. We also give examples of automatically presented groups.
\end{abstract}

Mathematics Subject Classification (2000). 20F69, 20F65, 20 E08.

Keywords. Property T, amenability, polycyclic group, nilpotent group, Grigorchuk topology, Cayley topology, groups generated by finite state automata, word problem, Haagerup property.

\section{Introduction}

We introduce the notion of an automatically presented group and the more general notion of a piecewise automatically presented group. Such a group is defined in a natural way by a finite state automaton (or a sequence of finite state automata in the general case) and admits the group generated by the finite state automaton (or, respectively, the piecewise automatic group defined by the sequence of automata) as a quotient. Informally speaking, a word in such a group is equivalent to the identity if it becomes trivial on some level of the tree on which the corresponding (piecewise) automatic group acts (see the next section for a formal definition).

Groups similar to automatically presented groups appear implicitly already in [14] (where Grigorchuk constructs torsion-free groups of intermediate growth). However, not much is known about this class of groups.

In this paper we provide examples of automatically presented groups. We show that, on one hand, these groups resemble groups generated by finite state automata in some aspects, and, on the other hand, these two classes of groups are basically different. We show, in particular, that an infinite piecewise automatically presented group cannot have the property $T$ of Kazhdan and that every finitely presented amenable group in this class is virtually abelian. This is in contrast with the fact that groups 
generated by finite state automata may have the property $T$ and that there are many solvable (e.g. nilpotent) finitely presented groups generated by finite state automata. We recall also that all known restrictions for a group to be generated by a finite state automaton are related to residual finiteness properties and properties of the word problem (see the last section of this paper for more details).

Finally, we show that piecewise automatic presentations help in establishing properties of some groups that were obtained earlier by other constructions.

\section{Finite state automata, automatically presented groups and piecewise automatically presented groups}

Definition 1. A finite state automaton over a finite alphabet $X$ consists of a finite set of states $A$ and a map $\tau: A \times X \rightarrow A \times X$.

Given a state $a$, denote by $\tau_{a}: X \rightarrow X$ the composition of $\tau(a,-)$ with the projection of $A \times X$ to $X$.

If, for every state $a \in A$, the map $\tau_{a}$ is bijective, the automaton is said to be invertible.

We recall the definition of a piecewise automatic group.

Definition 2. Consider an ascending sequence of sets $A_{1} \subseteq A_{2} \subseteq A_{3} \subseteq \cdots$ and a sequence of finite state invertible automata $\left(A_{n}, \tau_{n}\right), 1 \leq n<\infty$ defined over a common alphabet $X$ (an important example is $A_{1}=A_{2}=A_{3} \ldots$, with $\tau_{i}$ not necessarily equal to each other).

For every $a \in A_{1}$, define a transformation $\bar{a}$ of the set of onesided infinite words $X^{\infty}$ (or the set of finite words $X^{*}$ ) in the following way. Take $x=x_{1} x_{2} x_{3} \ldots$ in $X^{\infty}\left(\right.$ or in $\left.X^{*}\right)$. Set

$$
\left(a_{2}, y_{1}\right)=\tau_{1}\left(a, x_{1}\right)
$$

and, for all $j \geq 2$,

$$
\left(a_{j+1}, y_{j}\right)=\tau_{j}\left(a_{j}, x_{j}\right)
$$

Finally, define

$$
\bar{a}(x)=y_{1} y_{2} y_{3} \ldots
$$

Note that if all $\left(A_{n}, \tau_{n}\right)$ are invertible automata then, for every state $a \in A_{1}, \bar{a}$ is a bijection on $X^{\infty}$ (or $X^{*}$ ). Hence in this case we can consider the group generated by the transformations $\bar{a}, a \in A_{1}$. This group is called piecewise automatic group.

If all automata in the above definition are equal to some automaton $\tau$, we obtain the group generated by the finite state automaton $\tau$. 
Piecewise automatic groups were defined in [10] and [29] (in [10] we only considered the case when there is only a finite number of mutually distinct automata among $\tau_{n}$ ).

In the sequel we always assume that $A \subset e \cup S \cup S^{-1}, e \in A, \tau(e, x)=(e, x)$ and that $\tau\left(s^{-1}, y\right)=\left(a^{-1}, x\right)$ whenever $s, s^{-1} \in A$ and $\tau(s, x)=(a, y)$.

Consider an automaton $A, \tau$ over the alphabet $X$ and take a word $w$ over $A$. Note that $w$ defines a map $\tau_{w}: X \rightarrow X$. For $w=a_{1} a_{2} \ldots a_{k}, a_{i} \in A$,

$$
\tau_{w}=\tau_{a_{1}} \circ \tau_{a_{2}} \circ \cdots \circ \tau_{a_{k}}
$$

Here, as before, $\tau_{a_{i}}(x)$ is the projection of $\tau\left(a_{i}, x\right)$ onto $X$.

Let $\tau: A \times X \rightarrow A \times X$ be an invertible automaton and let $w$ be a word over the alphabet $A$. Note that, for every $x \in X, w$ defines a restriction $w_{x}$, which is again a word over $A$ : for $w=a_{1} a_{2} \ldots a_{k}, a_{i} \in A$,

$$
w_{x}=b_{1} b_{2} \ldots b_{k}
$$

where the state $b_{i}$ is the projection of $\tau\left(a_{i}, \tau_{a_{i+1} a_{i+2} \ldots a_{k}}(x)\right)$ onto $A$.

Also for a branch of the first level of the form $\gamma_{1}=x_{1} \ldots$ we say that $w_{x_{1}}$ is the restriction of $w$ to the branch $\gamma_{1}$ and for a branch $\gamma_{m}$ of level $m, \gamma_{m}=x_{1} \ldots x_{m} \ldots$, we define by induction on $m$ the restriction of $w$ to $\gamma_{m}$. This restriction is equal to the restriction to $x_{m}$ of the restriction of $w$ to the branch $\gamma_{m-1}=x_{1} \ldots x_{m-1} \ldots$ of level $m-1$.

Definition 3 (Automatically presented group). Let $\tau: A \times X \rightarrow A \times X(A \subset e \cup S \cup$ $S^{-1}, e \in A$ ) be an invertible automaton. The automatically presented group $G^{*}(\tau)$ over $\tau$ is the group given by the following presentation. The generating set is $S$ and a word $w$ over $S \cup S^{-1}$ is in the set of the defining relations if the following holds. There exists $i$ such that $w$ represents an element from the stabilizer of level $i$ (in the group generated by the finite state automaton $\tau$ ) and for every branch $\gamma$ of level $i$ the restriction of $w$ to $\gamma$ is freely equivalent to the identity in the group freely generated by $S$.

It is clear that the group generated by $\tau$ is a quotient of the automatically presented group over $\tau$. In the next section we see that in some cases these groups are equal and in some cases they are essentially different.

Given a sequence $A_{1} \subseteq A_{2} \subseteq A_{3} \subseteq \cdots$, a sequence of automata $\tau_{i}: A_{i} \times X \rightarrow$ $A_{i} \times X$ and a word in the alphabet $A_{1}$, we define the restrictions of $w$ to the branches of the rooted tree in a similar way as for a single automaton $\tau$. For a branch $\gamma_{m}$ of level $m, \gamma_{m}=x_{1} \ldots x_{m} \ldots$, we define by induction on $m$ the restriction of $w$ to $\gamma_{m}$. This restriction is equal to the restriction to $x_{m}$ with respect to the automaton $\tau_{m}$ of the restriction of $w$ to the branch $\gamma_{m-1}=x_{1} \ldots x_{m-1} \ldots$ of level $m-1$. By definition, the restriction of $w$ to a branch of level $m$ is a word over the alphabet $A_{m}$. 
Definition 4 (Piecewise automatically presented group). Consider an ascending sequence of sets $A_{1} \subseteq A_{2} \subseteq A_{3} \subseteq \cdots$ and a sequence of finite state invertible automata $A_{n}, \tau_{n}, 1 \leq n<\infty$, defined over a common alphabet $X$ (as before, an important case is $A_{1}=A_{2}=A_{3} \ldots$, with $\tau_{i}$ not necessarily equal to each other). We assume that $A_{i} \subset e \cup S_{i} \cup S_{i}^{-1}$ and that $e \in A_{i}$ for each $i$. The piecewise automatically presented group defined by the sequence $\left(A_{n}, \tau_{n}\right)$ is the group generated by $A_{1}$ in the following way. A word $w$ over $A_{1}$ is a defining relation in this group if there exists $i$ such that $w$ represents an element from the stabilizer of level $i$ (in the piecewise automatic group defined by $\tau_{1}, \tau_{2} \ldots$ ) and for every branch $\gamma$ of level $i$ the restriction of $w$ to $\gamma$ (the restriction is taken with respect to $\tau_{1}, \tau_{2}, \ldots, \tau_{i}$ ) is freely equivalent to the identity in the group freely generated by $S_{i}$.

If all $\left(A_{n}, \tau_{n}\right)$ coincide, then the piecewise automatically presented group defined above is equal to the automatically presented group over $\tau_{1}$.

It is clear that piecewise automatic groups are quotients of the corresponding piecewise automatically presented groups.

Note that, under the assumption of the previous definition, the piecewise automatically presented group is generated by $S_{1}$.

\section{Examples of automatically presented groups}

Example 1. Every finite group is automatically presented.

Indeed, let $G$ be a finite group, set $A=X=G$, and consider the automaton $\tau: A \times X \rightarrow A \times X$ given by $\tau\left(g_{1}, g_{2}\right)=\left(e, g_{1} g_{2}\right)$. It is clear that the automatically presented group over $\tau$ is equal to the group generated by this finite state automaton and is isomorphic to $G$.

Example 2. $\mathbb{Z}$ is automatically presented. Indeed, take any finite state automaton with a single non-identity state generating $\mathbb{Z}$. Such automata do exist: consider for example $\tau:\{a, e\} \times\{0,1\} \rightarrow\{a, e\} \times\{0,1\}$ defined by $\tau(a, 0)=(a, 1), \tau(a, 1)=(e, 0)$ and $\tau(e, x)=(e, x)$ for $x=0,1$. It is clear that the automatically presented group over $\tau$ is isomorphic to $\mathbb{Z}$.

Note that the class of automatically presented groups is closed under taking direct products. Indeed, let $G_{1}, G_{2}$ be automatically presented groups over $\tau_{1}$ and $\tau_{2}$, respectively, where $\tau_{1}: A_{1} \times X_{1} \rightarrow A_{1} \times X_{1}, \tau_{2}: A_{2} \times X_{2} \rightarrow A_{2} \times X_{2}, A_{1}=\bar{A}_{1} \sqcup e$, $A_{2}=\bar{A}_{2} \sqcup e$. Put $X=X_{1} \sqcup X_{2}, A=\bar{A}_{1} \sqcup \bar{A}_{2} \cup e$ and define $\tau: A \times X \rightarrow A \times X$ by $\tau(e, x)=(e, x)$, for all $x, \tau\left(a_{1}, x\right)=\tau_{1}\left(a_{1}, x\right)$ for $a_{1} \in \bar{A}_{1}, x \in X_{1}, \tau\left(a_{1}, x\right)=$ $(e, x)$ for $a_{1} \in \bar{A}_{1}, x \in X_{2}$ and similarly $\tau\left(a_{2}, x\right)=\tau_{2}\left(a_{2}, x\right)$ for $a_{2} \in \bar{A}_{2}, x \in X_{2}$, $\tau\left(a_{2}, x\right)=(e, x)$ for $a_{2} \in \bar{A}_{2}, x \in X_{1}$. It is clear that the automatically presented group over $\tau$ is $G_{1} \times G_{2}$.

Thus we see that, for every $n, \mathbb{Z}^{n}$ is automatically presented. 
Example 3. Let $\tau$ be the standard automaton generating the first Grigorchuk group. Recall that this is the finite state automaton $(A, \tau)$ over the alphabet $X=\{0,1\}$, where $A=\{a, b, c, d, e\}$ and $\tau: A \times X \rightarrow A \times X$ is defined by

$$
\tau(e, 0)=(e, 0), \quad \tau(e, 1)=(e, 1), \quad \tau(a, 0)=(e, 1), \quad \tau(a, 1)=(e, 0)
$$

and

$$
\begin{array}{lll}
\tau(b, 0)=(a, 0), & \tau(c, 0)=(a, 0), & \tau(d, 0)=(e, 0), \\
\tau(b, 1)=(c, 1), & \tau(c, 1)=(d, 1), & \tau(d, 1)=(b, 1) .
\end{array}
$$

Then the automatically presented group over $\tau$ contains a free non-abelian subgroup. In particular, this group is not equal to the group generated by the finite state automaton $\tau$.

Let us show that the subgroup generated by $b, c$ and $d$ is free. To see this take any word $w$ in $b, c, d$ representing a non-trivial element in the free group on these three generators. Observe that for every $n$ that is divisible by 3 the restriction of $w$ to the branch $11 \ldots 1 \ldots$ of level $n$ is again the word $w$. Therefore, $w$ represents a non-trivial element in the automatically presented group over $\tau$.

Example 4. Let $\tau$ be the automaton from [17], generating the Basilica group. Recall that this is the finite state automaton $(A, \tau)$ over the alphabet $X=\{0,1\}$, where $A=\{a, b, e\}$ and $\tau: A \times X \rightarrow A \times X$ is defined by

$$
\tau(a, 0)=(e, 0), \quad \tau(a, 1)=(b, 1), \quad \tau(b, 0)=(e, 1), \quad \tau(b, 1)=(a, 0),
$$

(and $\tau(e, x)=(e, x)$ for $x=0,1)$. We will prove bellow that the automatically presented group over $\tau$ is equal to the group generated by this automaton.

It was shown in [5] that the Basilica group is amenable. Therefore, we see that the class of automatically presented groups contains amenable groups that are not virtually abelian.

Lemma 1. (i) Let $w$ be a word of length $l, l \geq 3$, in the alphabet $a, b, a^{-1}, b^{-1}, e$ and let $\gamma$ be a branch of level 2 in the tree, corresponding to $X=\{0,1\}$ (that is, $\gamma$ is equal to $00 \ldots, 01 \ldots, 10 \ldots$ or $11 \ldots$ ). Then the restriction $w_{\gamma}$ of $w$ to $\gamma$ defined by $\tau$ (from Example 4) is freely equivalent to a word of length at most $l-1$.

(ii) Let $w$ be a word in the alphabet $a, b, a^{-1}, b^{-1}$, e of length 2 representing $a$ trivial word in the group, generated by the finite state automaton $\tau$. Then $w$ is freely equivalent to a trivial word.

Proof. (i) Note that for every automaton the restriction of a word of length $m$ to any branch has length at most $m$. Thus it is sufficient to consider the case $l=3$. Moreover, without loss of generality we can assume that $w$ is freely irreducible word (of length 3). 
First assume that $w$ contains as a subword one of the following words $b^{2}, b^{-2}, b a$, $b a^{-1}, a b^{-1}, a^{-1} b^{-1}$. Note that the restriction of each of these words to any branch of the first level is freely equivalent to a word of length at most 1 . Therefore, the restriction of $w$ to any branch of the first level, and consequently, to any branch of the second level has length at most $l-1$

Observe also that if $w$ contains as a subword $a^{2}$ or $a^{-2}$, then $w_{\gamma}$ has length at most $l-1$, because the restriction of $a^{2}$ and of $a^{-2}$ to any branch of level 2 is freely equivalent to a word of length at most 1 .

Therefore, it suffices to consider the case when $w$ is freely irreducible word of length 3 that does not contain as a subword any of the words $a^{2}, a^{-2}, b^{2}, b^{-2}, b a$, $b a^{-1}, a b^{-1}$, or $a^{-1} b^{-1}$. Note that in this case $w=b a b^{-1}$ or $b a^{-1} b^{-1}$. One can check that the restriction of each of these two words to any branch of the second level is freely equivalent to a word of length at most 2 .

(ii) It is sufficient to consider the case when $w$ is equal to $a^{2}, a^{-2}, a e, e a, b^{2}$, $b^{-2}$; Otherwise $w$ would either be freely equivalent to a trivial word, or its action of the first level would be non-trivial (and, therefore, $w$ would represent a non-trivial element in the group generated by the finite state automaton $\tau$ ). Each of the words $a^{2}, a^{-2}, a e, e a, b^{2}, b^{-2}$ acts non-trivially on the second level, and thus $w$ does not represent the trivial element in the group generated by $\tau$.

Note that the lemma above implies that the automatically presented group over $\tau$ (from Example 4) is equal to the group generated by this automaton. Indeed, take a word $w$ representing the identity in the group generated by $\tau$. The first part of the lemma implies that there exists a level $l$ such that each restriction of $w$ to a branch of this level has length at most two. Since $w$ represents the identity in the group generated by $\tau$, the action on the level $l$ of $w$ is trivial and all the restrictions represent the identity in the group generated by $\tau$. Combining this with the claim of the second part of the lemma, we see that each of these restrictions is freely equivalent to the identity word. Therefore, $w$ represents the identity element in the automatically presented group defined by $\tau$.

\section{Properties of piecewise automatically presented groups}

We recall that a finitely generated group $\Gamma$ has the property $T$ of Kazhdan if for some (and hence for all) finite generating set $S$ of $\Gamma$ there exists a positive constant $\varepsilon(S)$ such that for every unitary representation $(\pi, H)$ of $\Gamma$ with no invariant vectors and for every $u \in H$ there exists $s \in S$ such that $\|\pi(s) u-u\| \geq \varepsilon(S)\|u\|$. For more on the property $T$ see, for example, [20].

Theorem 1. Let $G$ be a piecewise automatically presented group.

(i) If $G$ has the property $T$ of Kazhdan, then $G$ is finite. 
(ii) If $G$ is amenable and finitely presented, then $G$ is virtually abelian.

(iii) More generally, if $G$ is a quotient of a finitely presented group $J$ without free subgroups, then $G$ is virtually abelian.

We recall that a group $\Gamma$ is polycyclic if there exists a sequence $e=\Gamma_{0} \subset \Gamma_{1} \subset$ $\cdots \subset \Gamma_{M}=\Gamma$ such that each $\Gamma_{i}$ is a normal subgroup in $\Gamma_{i+1}$ and $\Gamma_{i+1} / \Gamma_{i}$ is a (finite or infinite) cyclic group. It is known that every polycyclic group is finitely presented (see for example [25]). Therefore, the second part of the theorem shows in particular that every polycyclic piecewise automatically presented group is virtually abelian.

The theorem is in contrast with the fact that among groups generated by finite state automata there are groups having the property $T$ (see Mozes and Glasner, [22]) and there are many amenable finitely presented groups that are not virtually abelian (for example any nilpotent group admitting an expanding map is generated by some finite state automaton, [23]).

It is known that the Schreier graph of any contracting action on a regular rooted tree is of polynomial growth ([2], see also [23]), and thus groups admitting a faithful contracting action cannot have the property $T$ (unless they are finite). There is a conjecture that any group admitting a faithful contracting action is amenable. Note that we cannot replace the assumption in (i) by non-amenability.

Remark. The third part of the theorem implies that if $J$ is a group without freesubgroups admitting as a quotient the Basilica group from Example 3, then $J$ is infinitely presented.

Let $G$ be a finitely generated group and $S$ a finite generating set of $G$. Recall that the labeled Cayley graph of $(G, S)$ is the graph whose vertices are the elements of $G$ and in which two vertices $g_{1}$ and $g_{2}$ are joined by an edge whenever there exists $s \in S$ such that $g_{1} s=g_{2}$. In this case this oriented edge is labeled by $s$.

The Grigorchuk topology (also called the Cayley topology or the Chabauty topology) on the space of $d$-generated groups is the topology in which two groups $G_{1}$ and $G_{2}$ generated by $S,(\# S=d)$ are close whenever the labeled Cayley graphs of $\left(G_{1}, S\right)$ and $\left(G_{2}, S\right)$ coincide in the ball of radius $R$, for large $R$. This space is metrizable and the associated metric is $m_{G}\left(\left(G_{1}, S\right),\left(G_{2}, S\right)\right)=(1 / 2)^{R}$, where $R$ is the maximal radius such that the labeled Cayley graphs of $\left(G_{1}, S\right)$ and $\left(G_{2}, S\right)$ coincide in the ball of radius $R$. It is easy to see that the space of groups generated by a finite set $S$ is compact in this topology. See [19] for further properties of this topology.

Lemma 2. Let $G$ be the piecewise automatically presented group defined by the sequence $\left(A_{1}, \tau_{1}\right),\left(A_{2}, \tau_{2}\right), \ldots$ Consider a piecewise automatically presented group $G^{+}$, defined by the sequence $\left(A_{1}, \tau_{1}^{+}\right),\left(A_{2}, \tau_{2}^{+}\right), \ldots$, such that, for all $i \leq N$, we have $\tau_{i}=\tau_{i}^{+}$. 
If $N$ is large enough, then $G^{+}$is a quotient of some group $G^{\prime}$ which is close to $G$ in the Grigorchuk topology. That is, for every $\varepsilon>0$ there exists $N_{\varepsilon}$ and a group $G^{\prime}$, generated by $S$, satisfying $m_{G}\left(\left(G^{\prime}, S\right)(G, S)\right)<\varepsilon$ and such that $G^{+}$is a quotient of $G^{\prime}$, whenever $N>N_{\varepsilon}$. (Here in $G$ and $G^{+}$we consider the system of generators $S$ coming from $A_{1}$ ).

Proof. Let $R$ be a finite set of words representing the identity in $G$. Note that if $N$ is large enough, then by definition of piecewise automatically presented groups all words in $R$ represents the identity in $G^{+}$as well.

Now take $L>0$. Put $R_{L}$ to be the set of words of length at most $L$ (in the alphabet $A_{1}$ ) that represent the identity in the group $G$. Let $G^{\prime}$ be the group generated by $A_{1}$ with $R_{L}$ as the set of defining relations. By construction, the group $G^{\prime}$ is finitely presented. If $L$ is large enough, then $G^{\prime}$ is close to $G$ in the Grigorchuk topology. Note that there exists $N_{L}$ such that any group $G^{+}$satisfying the assumption of the lemma with $N>N_{L}$ is the quotient of the group $G^{\prime}$.

Proposition 1. Let $G$ be a piecewise automatically presented group. Suppose that $G$ $i$ is not virtually abelian. Then there exist sequences of groups $G_{i}, H_{i}$, such that, for every $i, H_{i}$ is a quotient of $G_{i}$, each $H_{i}$ contains a free non-abelian subgroup and $G_{i}$ tends to $G$ in the Grigorchuk topology.

Moreover, the sequences above can be chosen in such a way that $H_{i}$ admits a finite index subgroup which is a subgroup in a direct product of free groups.

Proof. Let $A_{i} \subset e \cup S_{i} \cup S_{i}^{-1}$. Let $\sigma_{i}$ be some automaton, such that the set of states of this automaton contains $S_{i} \cup S_{i}^{-1}$ and the subgroup generated by $S_{i}$ in the group generated by $\sigma_{i}$ (and hence in the automatically presented group over $\sigma_{i}$ as well) is free. (Such automata do exist, see e.g. [23], [24] or [28].)

Let $H_{i}$ be the piecewise automatically presented group defined at each level $j<i$ by the automaton for the level $j$ in the definition of $G$ and by $\sigma_{i}$ for $j \geq i$. Let us show that each $H_{i}$ contains a free group on two generators as a subgroup.

Indeed, for any level $i$ there are two words $w_{1}$ and $w_{2}$ over $S_{1}$ such that the action on this level defined by these words is trivial and such that for some branch $\gamma$ of level $i$ the restriction to this branch $w_{1}^{\gamma}$ and $w_{2}^{\gamma}$ represent two non-commuting words in the free group over $S_{i}$. (Otherwise the stabilizer of the level $i$ in $G$ would be abelian and $G$ would be virtually abelian.) We see that $w_{1}$ and $w_{2}$ freely generate a free subgroup in $H_{i}$.

Now, we apply Lemma 2 and see that there exists a sequence of groups $G_{i}$, such that, for every $i, H_{i}$ is a quotient of $G_{i}$ and $G_{i}$ tends to $G$ in Grigorchuk topology. (In this special case one can take $G^{\prime}=G^{+}$and it is not necessary to take a quotient, but we do not need it for the proof).

To prove the second claim of the proposition observe that the stabilizer of the level $i$ in $H_{i}$ is a subgroup in the direct product of free groups. Indeed, observe that 
a word in this stabilizer represents the identity in $H_{i}$ if and only if it represents the identity in the corresponding piecewise automatic group.

Proof of Theorem 1. To prove (i) we assume the contrary and suppose that $G$ is infinite and has the property $T$. Then $G$ is not virtually abelian. Therefore, we can apply Proposition 1 and conclude that there exist a sequence of groups $G_{i}$, tending to $G$ in the Grigorchuk topology, and a sequence of groups $H_{i}$ such that each $H_{i}$ is an infinite quotient of $G_{i}$, admitting a finite index subgroup $H_{i}^{\prime}$, which is a subgroup in a direct product of free groups.

We know that $G_{i}$ tends to $G$ and that $G$ has the property $T$. Recall that if $G$ has the property $T$ and $G^{\prime}$ is close enough to $H$ in the Grigorchuk topology, then $G^{\prime}$ also has the property $T$ ([26]). Therefore, there exists $N$ such that, for all $i \geq N, G_{i}$ has the property $T$. The property $T$ of Kazhdan is stable with respect to taking quotients, and therefore, for every $i \geq N$, the group $H_{i}$ has the property $T$. The property $T$ is also stable with respect to taking finite index subgroups, and so, for every $i \geq N, H_{i}^{\prime}$ has the property $T$.

On the other hand, free groups have the Haagerup property, which is stable with respect to taking direct products and subgroups (see e.g. [7]). Thus, for every $i$, the group $H_{i}^{\prime}$ has the Haagerup property.

In particular, $H_{N}^{\prime}$ is an infinite group that satisfies both the property $T$ and Haagerup property. We have arrived at a contradiction.

(ii) Since any group admitting a free non-abelian subgroup is non-amenable, (ii) is a particular case of (iii).

Now we prove (iii). We assume the contrary and suppose that $J$ is finitely presented group without free non-abelian subgroups and $G$ is a quotient of $J$. We suppose also that $G$ is not virtually abelian.

By Proposition 1 we know that there exists a sequence of groups $G_{i}$, tending to $G$ in the Grigorchuk topology, and a sequence of groups $H_{i}$ such that each $H_{i}$ contains a free non-abelian subgroup and such that $H_{i}$ is a quotient of $G_{i}$, for all $i$.

We know that $G_{i}$ tends to $G$ and that $G$ is a quotient of a finitely presented group $J$. Hence there exists $N$ such that, for every $i \geq N$, the group $G_{i}$ is a quotient of $J$. Since $J$ contains no free non-abelian subgroup and since this property is stable by taking a quotient, this implies that, for all $i \geq N, G_{i}$ contains no free non-abelian subgroups. Since each $H_{i}$ is a quotient of $G_{i}$, this shows that, for all $i \geq N, H_{i}$ contains no free non-abelian subgroups.

In particular, $H_{N}$ is a quotient of a group without free non-abelian subgroups, but $H_{N}$ itself contains a free non-abelian subgroup. We arrived at a contradiction. 


\section{Word problem}

The proof of the first part of the following proposition represents a well-known algorithm that solves the word problem in groups generated by finite state automata. The proof of the second part represents an algorithm that solves the word problem in automatically presented groups.

Proposition 2. (i) Every group $G$ generated by a finite state automaton is recursively presented and the word problem in such a group is solvable in exponential time: there exists an algorithm which, for a word $w$ of word length $l$, performs $\exp (C l)$ steps and decides whether $w$ represents the identity element in $G$.

(ii) Every automatically presented group $G$ (defined by a finite state automaton) is recursively presented and, moreover, the word problem in such a group is solvable in exponential time.

Proof. (i) Take $\tau: A \times X \rightarrow A \times X$ over an alphabet $X$ of cardinality $d$, and consider a word $w$ of length $l$ over the alphabet $A$ in the group $G$ generated by this finite state automaton.

Consider the action of $w$ on the first level of the tree. If it is non-trivial, the algorithm stops and answers that $w$ represents a non-identity element in $G$. If the action is trivial, consider the restrictions of $w$ to the branches of the first level. We denote these restrictions by $w_{1}, w_{2}, \ldots, w_{d}$.

Observe that the length of each $w_{i}$ is at most $l$. Apply recursively the same procedure to $w_{1}, \ldots, w_{d}$, having in mind that if, at some point, we get as a restriction a word $w$ that has already appeared, then we do not apply the algorithm to this additional occurrence of $w$. If at some point the algorithm stops producing new words (all restrictions of all considered words already appeared before) and all obtained words act trivially on the first level of the tree, then the algorithm stops and answers that $w$ represents the identity in $G$. Note that the algorithm makes at most $v_{G, A}(l)$ steps (where $v_{G, A}(l)$ is the growth function of $G$ with respect to the generating set $A$ ). At each step one performs at most $K l$ operations, where $K$ is some positive constant. Therefore, the total number of operations is at $\operatorname{most} \exp (C l)$, where $C$ is some positive constant.

(ii) Let $\tau: A \times X \rightarrow A \times X, A \subset S \cup S^{-1} \cup e$, be an automaton which gives an automatic presentation of $G$. Consider the set $W$ of non-empty freely irreducible words over the alphabet $S$ of length at most $l$. Consider the oriented graph $\Gamma_{W}$ whose vertices are the words in $W$ and in which two words $w_{1}$ and $w_{2}$ are joined by an edge in the direction from $w_{1}$ to $w_{2}$ if the restriction of $w_{1}$ to some branch of the first level is freely equivalent to $w_{2}$. In addition, draw a loop at every vertex $w$ that acts as a non-trivial permutation on the first level of the tree.

Observe that a word $w$ represents a non-identical element in the group $G$ if and only if there is an oriented path starting at $w$ and ending in a vertex which belongs to 
an oriented cycle of the graph $\Gamma_{W}$ (to see this we use again the fact that the restriction to a branch does not increase the length of the word).

For an oriented graph of bounded valency and of cardinality $s$ this problem can be solved in time, which is polynomial in $s$. Indeed, first we observe that we can determine in polynomial time which of the vertices belong to an oriented cycle. If a vertex belongs to some cycle, then it belongs to a cycle of length at most $s$. For every $m$ with $1 \leq m \leq s$ and for all pairs of vertices $w_{1}$ and $w_{2}$, we can determine whether there exists an oriented path of length $m$ joining these two vertices, and this can be done in polynomial time in $s$. To see this we apply an inductive argument and observe, that if we can do this for some $m<s$, then we can do it also for $m+1$. Indeed, $w_{1}$ and $w_{2}$ are joined by an oriented path of length $m+1$ if and only if there exists $w_{3}$ such that there is an oriented path of length $m$ from $w_{1}$ to $w_{3}$ and there is an oriented edge from $w_{3}$ to $w_{2}$. This shows, that we can determine which vertices belong to some oriented cycle in polynomial time in $s$. After this we take the vertex $w$ in our graph and for each vertex $u$ belonging to some oriented cycle we check whether there exists an oriented path of length at most $s$ from $w$ to $u$.

Now we return to the proof of (ii). Note that the cardinality of $\Gamma_{W}$ is at most $\exp (C l)$ and the valency of each vertex is bounded by the cardinality of $X$. Therefore, in time $\exp (K l)(K$ is a positive constant not depending on $l)$ we can determine which vertices correspond to a word, representing a non-identity element in $G$.

1) For a given automaton, it seems interesting to understand the structure of the graphs described in the proof of the second part of the proposition. In particular, additional information about these graphs can lead to better estimates for the word problem in the corresponding groups.

2) If the sequence of automata $\tau_{n}$ is recursive, then the piecewise automatically presented group defined by this sequence is recursively presented.

\section{References}

[1] L. Bartholdi, Endomorphic presentations of branch groups. J. Algebra 268 (2003), 419-443. Zbl 1044.20015 MR 2009317

[2] L. Bartholdi and R. I. Grigorchuk, On the spectrum of Hecke type operators related to some fractal groups. Trudy Mat. Inst. Steklova 231 (2000), 5-45; Proc. Steklov Inst. Math. 231 (2000), 1-41. Zbl 01729296 MR 1841750

[3] L. Bartholdi, R. I. Grigorchuk, and V. Nekrashevych, From fractal groups to fractal sets. In Fractals in Graz 2001, Trends Math., Birkhäuser, Basel 2003, 25-118. Zbl 1037.20040 MR 2091700

[4] L. Bartholdi and Z. Šunić, Some solvable automaton groups. In Topological and asymptotic aspects of group theory (Athens, OH, 2004), Contemp. Math. 394, Amer. Math. Soc., Providence, RI, 2006, 11-29. Zbl 05056623 MR 2216703 
[5] L. Bartholdi and B. Virág, Amenability via random walks. Duke Math. J. 130 (2005), 39-56. Zbl 05004322 MR 2176547

[6] T. Cecherini-Silberstein, R. I. Grigorchuk, and P. de la Harpe, Amenability and paradoxical decompositions for pseudogroups and discrete metric spaces. Trudy Mat. Inst. Steklova 224 (1999), 68-111; Proc. Steklov Inst. Math. 224 (1999), 57-97. Zbl 0968.43002 MR 1721355

[7] P.-A. Cherix, M. Cowling, P. Jolissaint, P. Julg, and A. Valette, Groups with the Haagerup property, Gromov's a-T-menability. Progr. Math. 197, Birkhäuser, Basel 2001. Zbl 1030.43002 MR 1852148

[8] A. Erschler, Boundary behavior for groups of subexponential growth. Ann. of Math. (2) 160 (2004), 1183-1210. Zbl 1089.20025 MR 2144977

[9] A. Erschler, On degrees of growth of finitely generated groups. Funktsional. Anal. $i$. Prilozhen. 39 (2005), 86-89; Funct. Anal. Appl. 39 (2005), 317-320. MR 2197519

[10] A. Erschler, Piecewise automatic groups. Duke Math. J. 134 (2006), 591-613.

[11] E. Følner, On groups with full Banach mean value. Math. Scand. 3 (1955), 243-244. Zbl 0067.01203 MR 0079220

[12] R. I. Grigorchuk, On Burnside's problem on periodic groups. Funktsional. Anal. $i$. Prilozhen. 14 (1980), 53-54; Funct. Anal. Appl. 14 (1980), 41-43. Zbl 0595.20029 MR 0565099

[13] R. I. Grigorchuk, Degrees of growth of finitely generated groups, and the theory of invariant means. Izv. Akad. Nauk SSSR Ser. Mat. 48 (1984), 939-985; Math. USSR-Izv. 25 (1985), 259-300. Zbl 0583.20023 MR 0764305

[14] R. I. Grigorchuk, On the growth degrees of $p$-groups and torsion-free groups. Mat. Sb. (N.S.) 126 (168) (1985), 194-214; Math. USSR-Sb. 54 (1986), 185-205. Zbl 0583.20024 MR 0784354

[15] R. I. Grigorchuk, Groups with intermediate growth function and their applications. Doctoral thesis, Moscow 1985.

[16] R. I. Grigorchuk and P. F. Kurchanov, Some questions of group theory related to geometry. In Algebra VII, Combinatorial group theory, Applications to geometry, Encyclopaedia Math. Sci. 58, Springer-Verlag, Berlin 1993, 167-232; translation from Itogi Nauki Tekh., Ser. Sovrem. Probl. Mat., Fundam. Napravleniya 58, 191-256 (1990). Zbl 0781.20023 MR 1265271

[17] R. I. Grigorchuk and A. Żuk, On a torsion-free weakly branch group defined by a three state automaton. Internat. J. Algebra Comput. 12 (2002), 223-246. Zbl 1070.20031 MR 1902367

[18] M. Gromov, Groups of polynomial growth and expanding maps. Inst. Hautes Études Sci. Publ. Math. 53 (1981), 53-73. Zbl 0474.20018 MR 0623534

[19] P. de la Harpe, Topics in geometric group theory. The University of Chicago Press, Chicago 2000. Zbl 0965.20025 MR 1786869

[20] P. de la Harpe and A. Valette, La propriété ( $T$ ) de Kazhdan pour les groupes localement compacts (avec un appendice de Marc Burger). Astérisque 175 (1989). Zbl 0759.22001 MR 1023471 
[21] V. A. Kaimanovich and A. M. Vershik, Random walks on discrete groups: boundary and entropy. Ann. Probab. 11 (1983), 457-490. Zbl 0641.60009 MR 0704539

[22] Ya. Glasner and Sh. Mozes, Automata and square complexes. Geom. Dedicata 111 (2005), 43-64. Zbl 1088.20037 MR 2155175

[23] V. Nekrashevych, Self-similar groups. Math. Surveys Monogr. 117, Amer. Math. Soc., Providence, RI, 2005. Zbl 1087.20032 MR 2162164

[24] A. S. Oliǐnyk, Free products of finite groups and groups of finitely automatic permutations. Trudy Mat. Inst. Steklova 231 (2000), 323-331; Proc. Steklov Inst. Math. 231 (2000), 308-315. Zbl 1005.20023 MR 1841761

[25] D. Segal, Polycyclic groups. Cambridge Tracts in Math. 82, Cambridge University Press, Cambridge 1983. Zbl 0516.20001 MR 0713786

[26] Y. Shalom, Rigidity of commensurators and irreducible lattices. Invent. Math. 141 (2000), 1-54. Zbl 0978.22010 MR 1767270

[27] A. M. Vershik, Amenability and approximation of infinite groups. Selecta Math. Soviet. 2 (1982), 311-330. Zbl 0533.22007 MR 0721030

[28] M. Vorobets and Ya. Vorobets, On a free group of transformations defined by an automaton. arXive:math.GR/0601231

[29] A. Woryna, On permutation groups generated by time-varying Mealy automata. Publ. Math. Debrecen 67 (2005), 115-130. Zbl 1081.20042 MR 2163119

Received April 10, 2006; revised October 8, 2006

Laboratoire de Mathématiques d'Orsay, UMR 8628 du C.N.R.S, Université Paris XI, 91405 Orsay, France

E-mail: anna.erschler@math.u-psud.fr; erschler@pdmi.ras.ru 